\title{
Therapeutic and prophylactic management of bleeding from oesophageal and gastric varices - recommendations of the Working Group of the National Consultant for Gastroenterology
}

\author{
Mariusz Rosołowski ${ }^{1}$, Marek Hartleb², Tomasz Marek², Janusz Milewski ${ }^{3}, K^{2}$ rzysztof Linke ${ }^{4}$, Grzegorz Wallner ${ }^{5}$, \\ Andrzej Dąbrowski ${ }^{1}$, Grażyna Rydzewska ${ }^{3,6}$ \\ ${ }^{1}$ Department of Gastroenterology and Internal Medicine, Medical University of Bialystok, Poland \\ ${ }^{2}$ Department of Gastroenterology and Hepatology, Medical University of Silesia, Katowice, Poland \\ ${ }^{3}$ Department of Internal Medicine and Gastroenterology, Central Clinical Hospital of Ministry of Internal Affairs, Warsaw, \\ Poland \\ ${ }^{4}$ Department of Gastroenterology, Human Nutrition and Internal Diseases, Poznan University of Medical Sciences, Poznan, \\ Poland \\ ${ }^{5}$ Department and Clinic of General Surgery, Gastroenterology and Digestive Tract Neoplasm, Medical University of Lublin, \\ Poland \\ ${ }^{6}$ Faculty of Sciences, University of Jan Kochanowski, Kielce, Poland
}

Key words: gastroesophageal varices, bleeding, management.

Address for correspondence: Mariusz Rosołowski MD, PhD, Department of Gastroenterology and Internal Medicine, Medical University of Białystok, 26a M. Skłodowska-Curie St, 15-276 Białystok, Poland, e-mail: Mariusz.Rosolowski@umb.edu.pl

\begin{abstract}
Gastroesophageal varices are one of the most serious consequences of portal hypertension. One-third of patients with varices will develop variceal haemorrhage. Despite significant improvements in the outcomes of treatment, mortality due to bleeding from gastro-oesophageal varices still remains very high. These recommendations present optimal management of patients with non-bleeding and bleeding varices.
\end{abstract}

\section{Introduction}

Portal hypertension in the course of liver cirrhosis is the cause of serious complications, including ascites, hepatic encephalopathy and bleeding from oesophageal varices. Oesophageal varices are one of the most serious consequences of portal hypertension; variceal bleeding is the cause of about one-third of deaths in patients diagnosed with cirrhosis of the liver. Despite significant improvement in the outcomes of both pharmacological and endoscopic treatment, 6-week mortality due to bleeding from oesophageal varices still remains at $10-20 \%$. Therefore, it seems reasonable to present recommendations for the optimal management of patients with bleeding and non-bleeding varices of the oesophagus/stomach, especially in the aspect of changing literature data and international guidelines [1, 2].

During discussions with the invited experts we established a common view on the definitions of the key terms associated with portal hypertension and oesophageal variceal bleeding, and we discussed the principles of modern diagnostics and therapy in these cases.

Oesophageal varices are the cause of $50-90 \%$ of cases of upper gastrointestinal tract bleeding in patients with liver cirrhosis, although other sources of bleeding should always be taken into account as well. Pending endoscopic confirmation of the bleeding site, due to the significantly worse prognosis of variceal bleeding compared to non-variceal bleeding, such cases should be treated as variceal, especially in the presence of significant haemodynamic symptoms. 


\section{Diagnosis of oesophageal varices}

For the diagnosis of oesophageal and gastric varices, endoscopy of the upper gastrointestinal tract should be performed. Other methods, including capsule endoscopy or endosonography, are not recommended as they are not fully reliable or costeffective.

\section{Comment}

In patients with a low risk of oesophageal varices, much attention is paid in the literature to mini-invasive or non-invasive methods of detection, which would allow for a possible reduction of the number of unnecessary endoscopies performed. The proposed methods refer to laboratory tests, including low platelet count, splenomegaly, platelet count, longitudinal spleen dimension $(\mathrm{mm})$ ratio over 909, portal vein diameter over $13 \mathrm{~mm}$, advanced liver cirrhosis according to the ChildPugh score, low prothrombin index and the degree of insulin resistance. The usefulness of non-invasive tests evaluating the degree of liver fibrosis, such as FibroTest or elastography, is also evaluated. The usefulness of endoscopic capsule for screening has also been reported. So far, however, none of these tests has proven to be sufficiently accurate; therefore, there is still a need to performendoscopy of the upper gastrointestinal tract in each patient at the time of diagnosis of liver cirrhosis. Endoscopic examination remains the "gold standard" for the diagnosis of oesophageal varices, and it permits their classification, which may be important in predicting first bleeding and/or recurrent bleeding. In addition to the size of varicose veins, the presence of so-called stigmata of imminent bleeding in the form of red or red-cherry points and/or streaks on the surface of the varicose columns is also important.

In the assessment of varicose veins, it is currently recommended to use a 3-point scale:

- small, simple, slightly raised above the level of the oesophagus,

- medium, tortuous, occupying less than one-third of the oesophageal lumen,

- large, occupying more than one-third of the oesophageal lumen.

\section{Comment}

Use of the above endoscopic classification of oesophageal varices permits standardisation of the descriptions of endoscopic examinations, and also is important for the prognosis of variceal bleeding. The current procedure for medium and large oesophageal varices is the same [1, 2]. As a result, there is an ongoing debate as to whether it would be reasonable, from a clinical point of view, to use a simple classification into small (with a diameter $<5 \mathrm{~mm}$ ) and large (with a diameter $>5 \mathrm{~mm}$ ) varicose veins.

When the presence of oesophageal varices has been confirmed, an important issue is to assess the risk of bleeding, and to determine active or recent variceal bleeding.

The stigmata of bleeding from oesophageal varices include:

- active variceal bleeding,

- "white nipple" lesions (white fibrin plug) on the varix,

- thrombus visible on the varix,

- no abnormalities which could be the source of bleeding other than oesophageal varices.

The management of a patient with oesophageal varices includes four main aspects that will be discussed in the present recommendations:

1. Prediction of the risk of variceal bleeding in patients with liver cirrhosis.

2. Prevention of first variceal bleeding.

3. Treatment of active variceal bleeding.

4. Prevention of recurrent variceal bleeding.

\section{Prediction of the risk of variceal bleeding in patients with liver cirrhosis}

The risk of bleeding in the absence of varicose veins on the first endoscopy in a patient with liver cirrhosis is low at about $2 \%$ per year. In the presence of small varicose veins, this risk increases to $5 \%$, and in the case of large ones, to $15 \%$ per year.

The risk factors for first oesophageal variceal bleeding include:

- variceal size (medium vs. large),

- stigmata of imminent bleeding on the variceal surface in the form of red streaks and points (even in the case of small varicose veins),

- advanced hepatic insufficiency (Child-Pugh class C), regardless of the variceal size,

- high portal pressure, or hepatic venous pressure gradient (HVPG) > $12 \mathrm{~mm} \mathrm{Hg}$.

The presence of at least one of these factors in an indication for use of primary prevention of variceal bleeding. 


\section{Comment}

Independent risk factors for bleeding from oesophageal varices also include viral aetiology or alcoholic liver cirrhosis.

All patients with liver cirrhosis should undergo endoscopy of the upper gastrointestinal tract for possible confirmation of the presence of oesophageal and/or gastric varices. Follow-up endoscopy is recommended in the following intervals:

- every 3 years in patients with compensated liver cirrhosis without identified varices,

- every 2 years in the case of small varicose veins,

- every year in the case of liver cirrhosis decompensation.

\section{Comment}

A number of studies point to the possible efficacy of other diagnostic methods, such as platelet count, spleen length, portal vein diameter or ultrasound measurement of liver stiffness (elastography). Unfortunately, the adequacy of these measurements in relation to the actual size of the varicose veins remains unsatisfactory. For this reason, endoscopy remains the "gold standard" for the diagnosis of variceal veins of the upper gastrointestinal tract. Follow-up examinations in patients with smal varicose veins should be performed to evaluate for the possibility of progression to large varicose veins. It has been suggested that follow-up endoscopy is not necessary after starting therapy with non-selective $\beta$-blockers.

\section{Prevention of first variceal bleeding}

It is not recommended to use non-selective $\beta$-blockers in the prevention of the formation of oesophageal varices in patients with liver cirrhosis. In the case of diagnosis of small oesophageal varices, non-selective $\beta$-blockers should be used only in patients with a high risk of bleeding. Patients with large varices of the oesophagus require primary prevention of bleeding with non-selective $\beta$-blockers.

\section{Comment}

Non-selective $\beta$-blockers used for primary prevention of bleeding from oesophageal varices include propranolol and nadolol (not available in Poland). Several studies also point to the beneficial effects of carvedilol, which has the additional $\alpha_{1}$-adrenolytic activity. So far, no significant advantage of any of these drugs has been shown. $\beta$-Blockers are currently not recommended for the prevention of varicose veins. Data from a large randomised clinical trial evaluating the effect of a non-selective $\beta$-blocker on the formation of oesophageal varices indicate a significant rate of adverse events and intolerance of these drugs in patients with liver cirrhosis [3]. Nonetheless, an approximately $10 \%$ reduction in portal venous pressure from baseline was associated with a reduced risk of varicose veins. The group of patients at high risk of bleeding from small varices includes patients with severe hepatic impairment (Child-Pugh class C) or with the presence of stigmata of imminent bleeding on the varicose columns [4]. A meta-analysis of studies on primary prevention of bleeding from large oesophageal varices clearly showed the efficacy of the above pharmacological treatment, which approximately halved the 2-year risk of bleeding. Hepatic venous pressure gradient value reduction of at least $10 \%$ relative to baseline in this group significantly reduces the risk of variceal bleeding, and HVPG values $<12 \mathrm{~mm} \mathrm{Hg}$ virtually eliminate the risk of first bleeding. Cyclic HVPG measurement, subject to the availability of this method, is the recommended procedure for monitoring the efficacy of treatment with a non-selective $\beta$-blocking agent.

The following methods are not recommended in the primary prevention of bleeding:

- nitrates,

- sclerotherapy,

- transjugular intrahepatic portosystemic shunt (TIPS).

\section{Comment}

Although in theory the use of nitrates, along with non-selective $\beta$-blockers, could increase the degree of portal venous pressure reduction, the significantly high number of side effects of this combination and the lack of evidence for the efficacy of this treatment from large randomised trials currently eliminates nitrates from the primary prevention of variceal bleeding. In addition, the significantly higher mortality seen in the group of patients over 50 years of age using nitrate monotherapy denies the clinical benefits of this management [5-7]. Vascular portosystemic shunts may be associated with a significantly increased risk of encephalopathy and higher mortality, so they are not recommended for primary prevention of bleeding $[8,9]$. Sclerotherapy of varicose veins in the primary prevention of bleeding is most likely associated with increased mortality in the above group of patients [10, 11].

Oesophageal variceal banding is the method recommended in patients with a high risk of first bleeding when non-selective $\beta$-blockers are not tolerated. 


\section{Comment}

The efficacy of endoscopic variceal banding has been proven in several randomised trials [12-14]. The findings suggest that banding is non-inferior, in terms of efficacy, to some non-selective $\beta$-blockers in the prevention of first bleeding. However, oesophageal variceal banding may be associated with an increased risk of bleeding from varices in the subcardiac area. It also seems that the risk of serious adverse events is lower when using $\beta$-blockers. The use of combination therapy in the primary prevention of variceal bleeding appears to be unfounded, as this management is associated with a significantly higher risk of adverse events [15].

\section{Treatment of active variceal bleeding}

The first procedure in a patient with variceal bleeding is an attempt to stabilise the cardiovascular system. In patients with a significant decrease in blood pressure, an attempt should be made to maintain mean blood pressure in the range of $80-100 \mathrm{~mm} \mathrm{Hg}$ and heart rate $\leq 100 \mathrm{bpm}$. When transfusing blood, the levels of haemoglobin and/ or haematocrit should not exceed 7-8 g/dl and $25 \%$, respectively.

\section{Comment}

Variceal bleeding is often massive, and haemodynamic destabilisation progresses rapidly. The results of studies suggest that actions aiming to obtain haemoglobin concentrations close to physiological levels are not recommended because they result in an increased risk of early recurrence of bleeding. However, in each case, when determining target haemoglobin concentrations, the patient's age, comorbidities and risk of recurrent bleeding should be taken into account.

Antibiotic prophylaxis should be used in every patient with liver cirrhosis and bleeding from the upper gastrointestinal tract. It is proposed that intravenous/oral fluoroquinolones or intravenous ceftriaxone are administered for short-term prophylaxis of bacterial infections (for up to 7 days).

\section{Comment}

Bacterial infections occur in approximately $20 \%$ of patients with liver cirrhosis and gastrointestinal bleeding. The number of infections may increase during hospitalisation. The most common infections are: spontaneous bacterial peritonitis, urinary tract infections and respira- tory tract infections [16]. The choice of antibiotic, and the optimal duration of its use, are not clearly defined. The results of studies suggest that patients with Child-Pugh class A hepatic impairment should receive fluoroquinolones, e.g. ciprofloxacin intravenously 400 mg/day. Patients with class $B$ or $C$ hepatic impairment should receive ceftriaxone 1-2 g/day. Antibiotic prophylaxis reduces the risk of recurrent bleeding and probably also mortality. It seems that patients with severe hepatic impairment benefit more from antibiotic use [17].

Vasoactive medications should be used after the onset of symptoms of bleeding from the upper gastrointestinal tract, with short-term continuation after endoscopic confirmation of the variceal source and effective endoscopic treatment of active variceal bleeding. The proposed duration of therapy is 3-5 days.

\section{Comment}

The results of numerous randomised trials suggest that medical therapy should be the first line of treatment for variceal bleeding. Somatostatin and terlipressin (both drugs available in Poland) have similar efficacy $[18,19]$. The choice of the product depends on the centre experience, economic aspects and the safety profile of a given therapy. According to the literature data, in elderly patients with a history of cardiovascular diseases, somatostatin has a slightly better safety profile. A very important factor affecting the possibility of stopping variceal bleeding is the effectiveness of the endoscopic method used. After stopping variceal bleeding, portal venous blood pressure suddenly increases. In the case of oesophageal variceal banding, portal pressure returns to baseline after approximately $48 \mathrm{~h}$, whereas after sclerotherapy it remains at a very high level for more than $120 \mathrm{~h}$. It seems, therefore, that the duration of drug treatment should take into account the type of endoscopic method used. The available data on the effect of using tissue adhesive for the treatment of variceal bleeding on portal venous pressure are limited. Currently, the optimal treatment for variceal bleeding is the simultaneous use of pharmacotherapy and one of the endoscopic procedures [20].

Endoscopic examination with possible invasive treatment should be performed as soon as possible (no later than $12 \mathrm{~h}$ from the moment of the patient's arrival at the hospital). In all cases, endotracheal intubation should be considered. 


\section{Comment}

The haemodynamic stability required before the decision can be made to start endoscopy for non-variceal bleeding is often impossible to obtain in the course of variceal bleeding. The procedure start time seems to have a significant impact on the effectiveness of endoscopic treatment. Therefore, it seems that the optimal time to start the endoscopic procedure is when the patient arrives at the hospital emergency department. Due to the significant risk of gastrointestinal content aspiration, haemodynamic instability and the unpredictable course of the endoscopic procedure, endotracheal intubation should always be considered [21].

Variceal banding is the preferred method of endoscopic treatment of oesophageal variceal bleeding. In the case of gastric variceal bleeding, the currently recommended method is tissue adhesive injections.

\section{Comment}

The available results of studies refer to treatment of variceal bleeding using banding, sclerosing agents or tissue adhesive. Banding of oesophageal varices is associated with the fewest complications, the highest efficacy in stopping bleeding and low mortality [22]. Gastric variceal bleeding is difficult to control using endoscopic methods. An effective method may be the use of a tissue adhesive [23]. The alternative is intravariceal administration of thrombin [24], the use of a Sengstaken-Blakemore tube or TIPS. There are ongoing studies evaluating the usefulness of a haemostatic powder (Hemospray) in the treatment of bleeding from oesophageal and/or gastric varices [25].

In case of failure of endoscopic therapy, temporary (for up to $24 \mathrm{~h}$ ) tamponade with a Sengstaken-Blakemore or equivalent tube can be performed, then the endoscopic procedure should be repeated. Transjugular intrahepatic portosystemic shunt or surgical treatment should be the treatment of choice in the above clinical settings.

\section{Comment}

In about $10-20 \%$ of cases, variceal bleeding cannot be stopped using endoscopic methods. In such cases, the preferred method is the use of a vascular polytetrafluoroethylene (PTFE)-coated, self-expanding TIPS stent between the hepatic vein and the intrahepatic segment of the portal vein. This method allows the cessation of bleeding in over $90 \%$ of cases [26]. An alternative ef- fective therapeutic method is the surgical formation of portosystemic anastomoses, especially when TIPS is not available. However, this procedure is associated with a mortality rate over $50 \%$, mainly due to perioperative complications, especially in patients with severe hepatic impairment [27]. Another highly effective emergency procedure is endoscopic placement of removable, self-expanding metal stents (SX-Ella Danis stent). The stent is removed without significant complications after 7-14 days [28]. Liver transplantation should be considered in patients who are eligible for such treatment.

\section{Prevention of recurrent variceal bleeding}

The secondary prevention of oesophageal variceal bleeding should involve treatment with non-selective $\beta$-blockers combined with endoscopic variceal banding. Variceal banding procedures must be performed until complete eradication, approximately every 2 weeks (1-4 weeks).

\section{Comment}

Patients who have survived variceal bleeding are at high risk of recurrent bleeding. Without prevention, the recurrence rate is approximately $60 \%$ within $1-2$ years, with a mortality rate of about $30 \%$. Non-selective $\beta$-blockers at a maximum but well tolerated dose are moderately effective in the secondary prevention of bleeding from oesophageal varices. Any additional use of nitrates may be synergistic, but the significant number of side effects of this method often does prevents the use of combination therapy. Patients in whom HVPG can be reduced by at least $20 \%$ from baseline or to $<12 \mathrm{~mm} \mathrm{Hg}$ are at the lowest risk of recurrent bleeding. Banding of oesophageal varices is the endoscopic treatment of choice in the secondary prevention of oesophageal variceal bleeding. The number of procedures usually includes 3-4 sessions of banding. The results of studies comparing the efficacy of oesophageal variceal banding and optimal pharmacological therapy are inconclusive, suggesting the equivalence of the two methods. The optimal procedure is the combination of endoscopic variceal banding with medical therapy [29]. Monotherapy with non-selective $\beta$-blockers is probably ineffective in the secondary prevention of gastric variceal bleeding [30].

Sclerotherapy and combined use of endoscopic banding and sclerotherapy are not recommended in the secondary prevention of oesophageal variceal bleeding. 


\section{Comment}

The results of meta-analyses of multiple randomised trials have shown that endoscopic variceal banding is more effective than sclerotherapy in the prevention of recurrent bleeding from oesophageal varices. In addition, banding was associated with a significantly lower risk of complications. Also, meta-analyses of studies have not shown any benefit from the additional use of sclerotherapy; however, a higher rate of clinically significant oesophageal stenosis was seen after this procedure [31].

\section{References}

1. Garcia-Tsao G, Sanyal AJ, Grace ND, Carey W. Prevention and management of gastroesophageal varices and variceal hemorrhage in cirrhosis. Hepatology 2007; 46: 922-38.

2. de Franchis R. Revising consensus in portal hypertension: report of the Baveno $V$ consensus workshop on methodology of diagnosis and therapy in portal hypertension. J Hepatol 2010; 53: 762-8.

3. Groszmann RJ, Garcia-Tsao G, Bosch J, et al. Beta-blockers to prevent gastroesophageal varices in patients with cirrhosis. N Engl J Med 2005; 353: 2254-61.

4. Merkel C, Marin R, Angeli P, et al. A placebo-controlled clinical trial of nadolol in the prophylaxis of growth of small esophageal varices in cirrhosis. Gastroenterology 2004; 127: 476-84.

5. Borroni G, Salerno F, Cazzaniga M, et al. Nadolol is superior to isosorbide mononitrate for the prevention of the first variceal bleeding in cirrhotic patients with ascites. J Hepatol 2002; 37: 315-21.

6. Garcia-Pagan JC, Villanueva C, Vila MC, et al. Isosorbide mononitrate in the prevention of first variceal bleed in patients who cannot receive beta-blockers. Gastroenterology 2001; 121: 908-14.

7. Angelico M, Carli L, Piat C, et al. Effects of isosorbide-5-mononitrate compared with propranolol on first bleeding and long-term survival in cirrhosis. Gastroenterology 1997; 113: 1632-9.

8. Conn HO, Lindenmuth WW, May CJ, Ramsby GR. Prophylactic portacaval anastomosis. Medicine (Baltimore) 1972; 51: 27-40.

9. Sanyal AJ, Freedman AM, Shiffman ML, et al. Portosystemic encephalopathy after transjugular intrahepatic portosystemic shunt: results of a prospective controlled study. Hepatology 1994; 20: 46-55.

10. Avgerinos A, Armonis A, Manolakopoulos S, et al. Endoscopic sclerotherapy plus propranolol versus propranolol alone in the primary prevention of bleeding in high risk cirrhotic patients with esophageal varices: a prospective multicenter randomized trial. Gastrointest Endosc 2000; 51: 652-8.

11. Prophylactic sclerotherapy for esophageal varices in men with alcoholic liver disease. A randomized, single-blind, multicenter clinical trial. The Veterans Affairs Cooperative Variceal Sclerotherapy Group. N Engl J Med 1991; 324: 1779-84.

12. Schepke M, Kleber G, Nurnberg D, et al. Ligation versus propranolol for the primary prophylaxis of variceal bleeding in cirrhosis. Hepatology 2004; 40: 65-72.

13. Drastich P, Lata J, Petrtyl J, et al. Endoscopic variceal band ligation compared with propranolol for prophylaxis of first variceal bleeding. Ann Hepatol 2011; 10: 142-9.

14. Perez-Ayuso RM, Valderrama S, Espinoza M, et al. Endoscopic band ligation versus propranolol for the primary prophylaxis of variceal bleeding in cirrhotic patients with high risk esophageal varices. Ann Hepatol 2010; 9: 15-22.
15. Lo GH, Chen WC, Wang HM, Lee CC. Controlled trial of ligation plus nadolol versus nadolol alone for the prevention of first variceal bleeding. Hepatology 2010; 52: 230-7.

16. Soares-Weiser K, Brezis M, Tur-Kaspa R, Leibovici L Antibiotic prophylaxis for cirrhotic patients with gastrointestinal bleeding. Cochrane Database Syst Rev 2002; (2): CD002907.

17. Hou MC, Lin HC, Liu TT, et al. Antibiotic prophylaxis after endoscopic therapy prevents rebleeding in acute variceal hemorrhage: a randomized trial. Hepatology 2004; 39: 746-53.

18. Ioannou G, Doust J, Rockey DC. Terlipressin for acute esophageal variceal hemorrhage. Cochrane Database Syst Rev 2003; (1): CD002147.

19. Valenzuela JE, Schubert T, Fogel MR, et al. A multicenter, randomized, double-blind trial of somatostatin in the management of acute hemorrhage from esophageal varices. Hepatology 1989; 10: 958-61.

20. Banares R, Albillos A, Rincon D, et al. Endoscopic treatment versus endoscopic plus pharmacologic treatment for acute variceal bleeding: a meta-analysis. Hepatology 2002; 35: 609-15.

21. Rudolph SJ, Landsverk BK, Freeman ML. Endotracheal intubation for airway protection during endoscopy for severe upper GI hemorrhage. Gastrointest Endosc 2003; 57: 58-61.

22. Stiegmann GV, Goff JS, Michaletz-Onody PA, et al. Endoscopic sclerotherapy as compared with endoscopic ligation for bleeding esophageal varices. N Engl J Med 1992; 326: 1527-32.

23. Romero-Castro R, Ellrichmann M, Ortiz-Moyano C, et al. EUS-guided coil versus cyanoacrylate therapy for the treatment of gastric varices: a multicenter study (with videos). Gastrointest Endosc 2013; 78: 711-21.

24. Ramesh J, Limdi JK, Sharma V, Makin AJ. The use of thrombin injections in the management of bleeding gastric varices: a single-center experience. Gastrointest Endosc 2008; 68: 877-82.

25. Stanley AJ, Smith LA, Morris AJ. Use of hemostatic powder (Hemospray) in the management of refractory gastric variceal hemorrhage. Endoscopy 2013; 45 Suppl 2 UCTN: E86-7.

26. Sanyal AJ, Freedman AM, Luketic VA, et al. Transjugular intrahepatic portosystemic shunts for patients with active variceal hemorrhage unresponsive to sclerotherapy. Gastroenterology 1996; 111: 138-46.

27. Rikkers LF, Jin G, Burnett DA, et al. Shunt surgery versus endoscopic sclerotherapy for variceal hemorrhage: late results of a randomized trial. Am J Surg 1993; 165: 27-32.

28. Wright $\mathrm{G}$, Lewis $\mathrm{H}$, Hogan $\mathrm{B}$, et al. A self-expanding metal stent for complicated variceal hemorrhage: experience at a single center. Gastrointest Endosc 2010; 71: 71-8.

29. Thiele M, Krag A, Rohde U, Gluud LL. Meta-analysis: banding ligation and medical interventions for the prevention of rebleeding from oesophageal varices. Aliment Pharmacol Ther 2012; 35 : 1155-65.

30. Hung HH, Chang CJ, Hou MC, et al. Efficacy of non-selective beta-blockers as adjunct to endoscopic prophylactic treatment for gastric variceal bleeding: a randomized controlled trial. J Hepatol 2012; 56: 1025-32.

31. Saeed ZA, Stiegmann GV, Ramirez FC, et al. Endoscopic variceal ligation is superior to combined ligation and sclerotherapy for esophageal varices: a multicenter prospective randomized trial. Hepatology 1997; 25: 71-4.

Received: $\quad 15.04 .2014$

Accepted: 17.04 .2014 